as median-high risk (table 1). The sensitivities and specificities are as follows for each tool: QRISK2 (19\%, 93\%), FRS (22\%, 93\%), mFRS (46\%, 83\%), QRISK3 (47\%, 78\%), SLECRE $(61 \%, 63 \%)$, respectively. The tools were similar in negative predictive value, ranging from $89 \%$ (QRISK2) to $92 \%$ (SLECRE). The FRS and mFRS had the greatest c-statistics, both equalling 0.73 , demonstrating the greatest predictive accuracy amongst the tools, while the QRISK3 and SLECRE had the lowest (0.67).

Conclusions While the mFRS performance was superior to the FRS, the QRISK3 did not outperform the mFRS. Although the SLECRE had the highest sensitivity, it had the lowest specificity, demonstrated by grouping the most cases and controls in the median-high risk category. Several factors are important to consider when deciding which risk assessment tools to utilize clinically: ease of use, sensitivity/specificity, and laboratory data accessibility. Thus, the mFRS continues to be a practical tool with a simple, intuitive scoring system appropriate for the ambulatory clinic setting based on the initial weighting of the FRS while adjusting for SLE.

Funding Source(s): Lupus Foundation of America - Gina M. Finzi Memorial Student Summer Fellowship

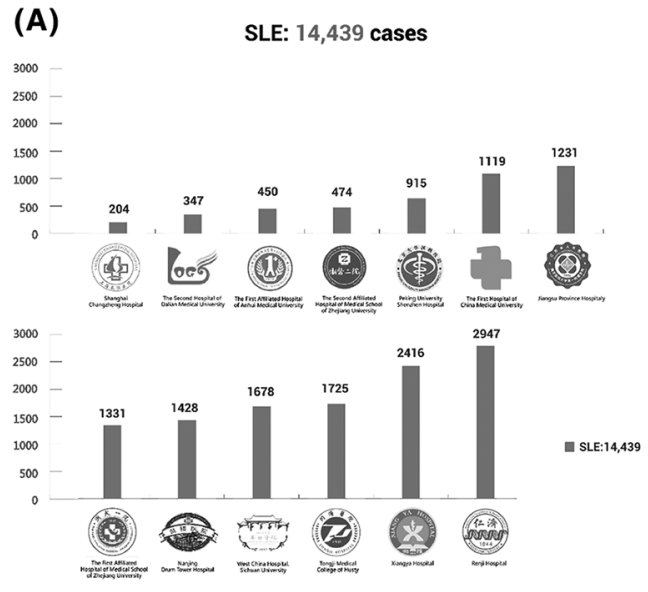

(C)

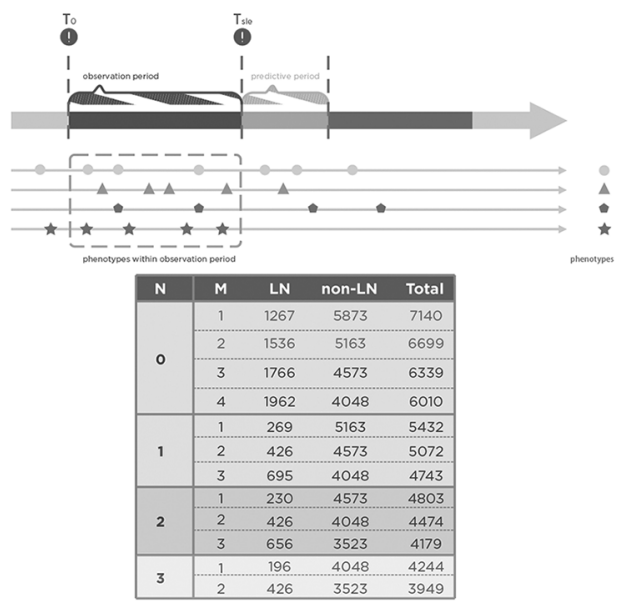

Cardiovascular Disease Risk Stratification by Risk Assessment Tool

\section{ARTIFICIAL INTELLIGENCE PREDICT THE LUPUS NEPHRITIS BASED ON FULL-PHENOTYPE DATABASE WITH NATURAL LANGUAGE PROCESSING TECHNOLOGY}

${ }^{1}$ Ting Li*, ${ }^{2}$ Lei Jiang, ${ }^{3}$ Li Wang, ${ }^{4}$ Chunde Bao, ${ }^{5}$ Huji Xu. ${ }^{1}$ Shanghai Jiaotong University School of Medicine affiliated Renji Hospital; ${ }^{2}$ Department of Rheumatology and Immunology, Changzheng Hospital, Second Military Medical UniversityShanghai200433China; ${ }^{3}$ Department of medical informatics, Medical School, Nantong University, 19 Qixiu Road, 226001 Nantong, Jiangsu Province, China; ${ }^{4}$ Renji Hospital, School of Medicine, Shanghai Jiao Tong University, China; ${ }^{5}$ The Second Military Medical University

\subsection{6/lupus-2019-Ism.292}

Background As the prototype of autoimmune disease, systemic lupus erythematosus (SLE) has complex and diverse clinical manifestations which may be harmful even life-threaten. Its pitiful that we can just passively respond to these serious complications. It will be a great advantage if the high-risk groups

(B)
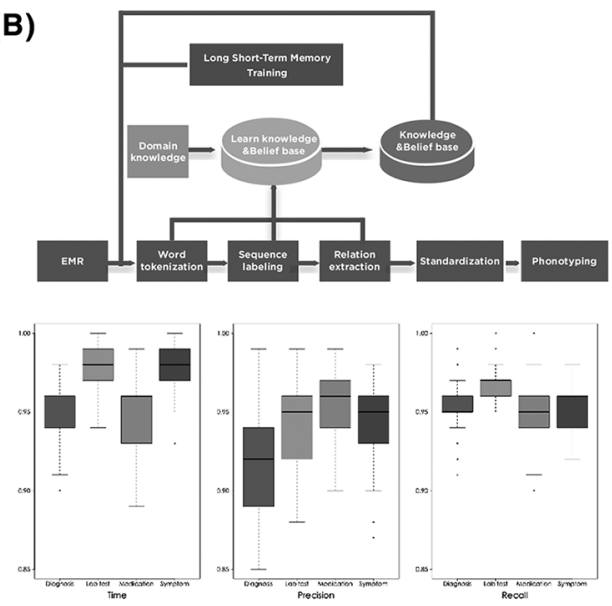

(D)

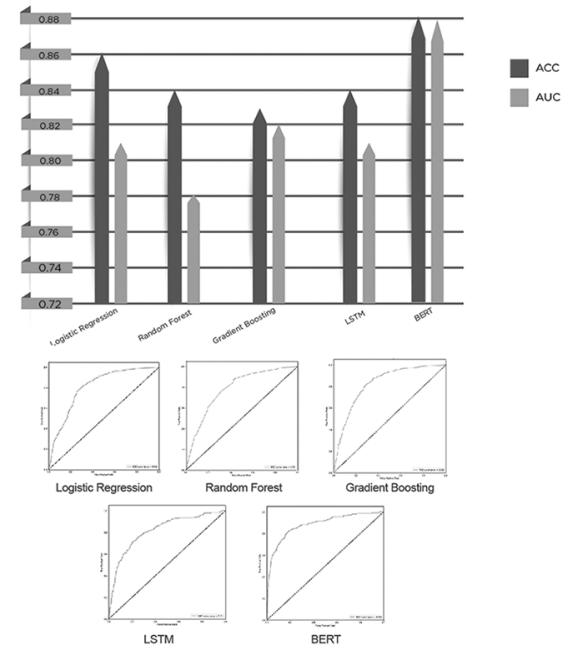

SLE (Systemic lupus Erythematosus) ～LSTM (Long Short-Term Memory) $\quad$ LN (lupus Nephritis) B BERT(Bidirectional Encoder Representations from Transformers)

$\mathrm{Ni}, i \in[0,3]: \mathrm{N}_{0}$ is that the patient has a record in the EMR until the first SLEdiagnosis and $\mathrm{N}_{i+1}$ for every 360 days. $M t, t \in[1,3]: M_{1}$ refer to the 1 year period after SLE being diagnosed and $M_{t+1}$ for every 360 days. 
could be predicated and prevented with pre-treatment. The raising of risk prediction models depends on the collection of patient phenotypes, which are scattered in various forms and very cumbersome.

In this study, we collected the largest database of complete medical record of inpatients of lupus in China. The clinical phenotype database was generated by using natural language processing (NLP) techniques, then lupus nephritis (LN) prediction model was built.

Methods A total of 14,439 SLE patients were collected from the rheumatology and immunology departments of 13 Chinese tertiary hospitals in this study, including 13062 females (90.46\%), with an average age of 33.4 years, and the time span of EMR (Electronic Medical Records) was from October 28, 2001 to March 31, 2017. It includes basic information about patients, physical examination, inspection and diagnostic information, etc. We designed a hybrid NLP system combined NLP technical and expert knowledge at the same time, which was named as Deep Phenotyping System (DPS), to extract all the phenotypic information recorded in EMR. Based on these standard formatted entities, the machine learning and deep learning prediction methods are used to predict the LN in SLE.

Results The DPS efficiently processed EMR data, and its accuracy, precision, and recall were each greater than 93\%. It extracted 73794 entities from 14,439 SLE cases, each with time attributes, and produced 18,785,000,640 entities. Thus, a LN prediction model was raised, which the likelihood of lupus patients without nephritis will develop lupus nephritis within half and one year can be predicted.) More than 35000 phenotypes was used in this model and it was verified with independent samples. The best accuracyACC and area under the curve (AUC) can be achieved 0.88 and 0.86 respectively.

Conclusions The comprehensive SLE phenotype database constructed by NLP greatly improves the research efficiency of lupus clinical phenotype. We first proposed a predictive model of lupus nephritis, which is high applicability and efficiency. The experimental results of good close and open testing fully demonstrate the authenticity and practicality of this database. The research process and method based on real world data are also applicable to predict other important complications of lupus.

Funding Source(s): None

\section{A NOVEL CD3/BCMA BISPECIFIC ANTIBODY SELECTIVELY KILLS PLASMA CELLS IN BONE MARROW OF HEALTHY INDIVIDUALS WITH IMPROVED SAFETY}

${ }^{1}$ Wim van Schooten*, ${ }^{2}$ Kerstin Juelke, ${ }^{3}$ Suhasini lyer, ${ }^{1}$ Ben Buelow, ${ }^{1}$ Roland Buelow, ${ }^{4}$ Dieter Volk. ${ }^{1}$ Teneobio, Inc; ${ }^{2}$ Institute for Medical Immunology, Charité University of Medicine; ${ }^{3}$ Teneobio; ${ }^{4}$ Institute for Medical Immunology, Charité University of Medicine, Berlin, Germany

\subsection{6/lupus-2019-Ism.293}

Background Autoantibodies play an important role in the pathogenesis of systemic lupus erythematosus (SLE). Plasma cells secrete these autoantibodies but are unfortunately refractory to conventional immunosuppressive treatments. Bcell Maturation Antigen (BCMA) is exclusively expressed on mature B cells and, for that reason, is considered a good target for plasma cell depletion. Here, we analyze a promising therapeutic in development for Multiple Myeloma (MM), that could be used for the treatment for SLE. The antiCD3*BCMA bispecific antibody TNB-383B is in clinical trials for the treatment of MM. In vitro experiment showed that TNB-383B added to human bone marrow samples ex vivo induced a dose dependent lysis of BCMA-expressing plasma cells.

Methods Depletion of plasma cells by TNB-383B was tested using bone marrow samples extracted from bone retrieved of hip replacments. Human bone marrow mononuclear cells $(n=10)$ were incubated with TNB-383B at increasing concentrations. The activity of TNB-383B was compared to a positive control antibody (PC), which has a BCMA-binding domain and an $\mathrm{Fc}$ region identical to $\mathrm{TNB}-383 \mathrm{~B}$, but binds to CD3 with stronger affinity. Bone marrow mononuclear cells were incubated for 18 hours with controls and TNB383B and samples were analyzed for plasma cell depletion (cells expressing CD19+, IgD-, IgM-, CD38++, CD27 ++ ) and $\mathrm{T}$ cell activation (CD69, CD107a, CD137, CD154). Cytokine production (IFN-, TNF, IL-2, IL-6, IL12p70, IL-13, IL-4 and IL-10) was evaluated using multiplex.

Results TNB-383B effectively depleted plasma cells in bone marrow samples of healthy individuals undergoing hip replacement; more than $80 \%$ of BCMA expressing plasma cells were depleted after an overnight incubation with TNB-383B or a positive control. Analysis activation markers showed that TNB-383B activated T cells as evidenced by the expression of activation markers (CD69, CD154 or CD137) but only minimal cytokine production was observed.

Conclusions TNB-383B represents a novel immunotherapeutic with improved safety and efficacy for the treatment of pathogenic long-lived plasma cells. TNB-383B is in clinical trials for the treatment of Multiple Myeloma. TNB-383B could be an attractive treatment to prevent acute organ rejection in Panel Reactive Antibody (PRA) organ transplantation and autoimmune patients including Systemic Lupus Erythematosis. Teneobio anticipates to start testing TNB-383B in PRA patients by late 2019.

Funding Source(s): Teneobio, Inc.

\section{IMMUNOPHENOTYPIC SUBGROUPS OF SLE DEFINED BY AUTOANTIBODIES, GENE EXPRESSION AND FLOW CYTOMETRIC ANALYSIS}

${ }^{1}$ Marta Aguilar Zamora*, ${ }^{2} \mathrm{Hui}$ Lu, ${ }^{2}$ Danynag Li, ${ }^{2}$ Zoe Betteridge, ${ }^{3}$ Katie Dutton, ${ }^{3} \mathrm{Md}$ Yuzaiful Md Yusof, ${ }^{3}$ Antonios Psarras, The MASTERPLANS Consortium, ${ }^{4}$ Ian N Bruce, ${ }^{2}$ Neil McHugh, ${ }^{3}$ Edward Vital. ${ }^{1}$ Hospital Universitario Dr. Peset, Valencia. Fundación Valenciana de Reumatologia; ${ }^{2}$ University of Bath; ${ }^{3}$ University of Leeds; ${ }^{4}$ University of Manchester

\subsection{6/lupus-2019-|sm.294}

Background SLE may be stratified according to a range of different immune assessments but the relationships between these are less well defined. MASTERPLANS is an MRC-funded consortium that seeks to identify immunophenotypic subgroups of patients that predict response to therapy. Our objective here was to analyse a clinically well-phenotyped patients using a suite of immune assessments and identify inter-relationships 